\title{
Assessment of Knowledge of Oral Hygiene Aids among Dentists
}

\author{
Keerththana Balabaskaran ${ }^{1}$, Dr.Jai Ganesh Ramamurthy ${ }^{2}$ \\ ${ }^{\prime}$ (Saveetha Dental college, India) \\ ${ }^{2}$ (Department of Periodontics, Saveetha Dental College, India)
}

\begin{abstract}
Objectives: The present study was carried out to assess the knowledge of oral hygiene aids among practicing general dentists.

Materials and methods: A 14 multiple choice questionnaire was given to practicing general dentists to answer. A total of 50 dentists participated in the survey (26 females and 24 males).

Results: $74 \%$ advises to brush twice, $70 \%$ in the morning and night. $82 \%$ demonstrate their patients to use dental floss, $62 \%$ use disclosing agent to motivate the patient. $58 \%$ size of the pea tooth paste. $46 \%$ recommend the use of brush and paste for cleaning the teeth. 90\% recommend the use of inter dental brushes during orthodontic treatment. About $46 \%$ recommend their patients to replace their tooth, when bristles begin to fray. $64 \%$ answered horizontal scrub causes cervical abrasion and gingival recession. $44 \%$ answered modified stillman is used in gingival recession and root exposure. $42 \%$ answered charters in cleaning the areas of healing wounds after periodontal surgery. 50\% answered that Unitufted brushes are used in type 3 embrasure $.60 \%$ answered that chlorhexidine is a Bisquanides. About $66 \%$ answered that strontium salt is the desensitizing agent used in tooth paste.

Conclusion: The present study shows better knowledge and awareness of oral hygiene aids among dental practitioners. For optimum plaque control, the clinician needs to be working closely with the patient to communicate clear and simple messages and tailor the oral hygiene aids to their needs.

Key words: brushing techniques, chlorhexidine, dental flossing, oral hygiene aids, tooth brush.
\end{abstract}

\section{Introduction}

Oral health care practices have been proved to be an effective preventive measure for maintaining good oral health as part of general health ${ }^{[1]}$. The use of oral hygiene aids may improve tooth cleaning effectiveness provided that cleaning is sufficiently through and performed at appropriate intervals ${ }^{3}$. Several studies have shown the usefulness of regular dental flossing for removing interdental plaque and calculus formation ${ }^{2}$. Optimum plaque control can be achieved with a combination of physical plaque removal and chemotherapeutic agents and the risk of dental disease reduced.

\section{Materials And Methods:}

A questionnaire of 14 multiple choice questions were given to general dental practitioners. A total of 50 dentists participated in the study of which $52 \%$ are females and $48 \%$ are males. The questions were about their oral hygiene practices, their awareness about oral hygiene aids, brushing techniques.

\section{Questions}

1) How many times a day you recommend cleaning of teeth?

a) Once daily

b) Twice

c) Thrice

d) After every meal

e) Others

2) When?

a) Morning

b) Evening

c) Night

3) Do you demonstrate your patients to use dental floss?

a) Yes

b) No

4) Do you use disclosing gent to motivate the patient?

a) Yes

b) No

5) How much tooth paste do you recommend? 
a) Half length of a brush

b) Full length of a brush

c) Size of a pea

d) Size of grain rice

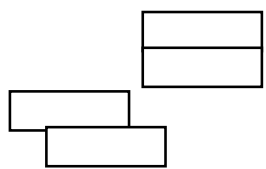

6) What do you recommend commonly for cleaning teeth?

a) Brush \& paste

b) Brush \&paste + dental floss

c) Brush \& paste + dental floss + mouth wash

7) Do you recommend the use of inter dental brushes during orthodontic treatment?

a) Yes

b) No

8) When do you recommend your patients to replace their tooth brush?

a) Once in 6 months

b) When the bristles begins to fray

c) When the color of the bristles begin to change

d) Once in a year

9) Which technique causes cervical abrasion and gingival recession?

a) Charters

b) Modified stillman

c) Horizontal scrub method

d) Bass

10) Which tooth brushing techniques you recommend in gingival recession and root exposure?

a) Bass

b) Modified stillman

c) Modified bass

d) Fones

11) What tooth brushing technique is recommended in cleaning the areas of healing wounds after periodontal surgery?

a) Bass

b) Modified stillman

c) Charters

d) Horizontal

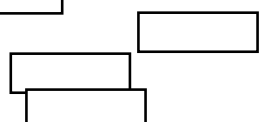

12) In type 3 embrasure, the interdental aid selected is

a) Dental floss

b) Unitufted brushes

c) Miniature interdental aids

13) Chlorhexidine is a

a) Metallic salts

b) Quaternary ammonium compounds

c) Bisquanides

d) Phenolic compounds

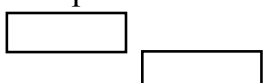

14) The desensitizing agent used in tooth paste is

a) Carboxy-methyl cellulose

b) Benzoic acid

c) Strontium salts

d) Calcium carbonate

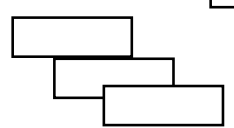

\section{Results:}

The results are summarizing responses to each 14 questions in the survey.

1) How many times a day you recommend cleaning of teeth?

Among 50 dentists, $16 \%$ recommends the cleaning of teeth once daily, $74 \%$ advise to brush twice, $8 \%$ recommend cleaning after every meals, $2 \%$ prefer others. (fig 1 )

2) When?

Among 50 dentists about $26 \%$ recommends brushing in the morning, $4 \%$ in the evening and $70 \%$ in the morning and night.

3) Do you demonstrate your patients to use dental floss? 
Among 50 dentists about $82 \%$ demonstrate their patients to use dental floss, $18 \%$ does not demonstrate the use of dental floss.( Table A)

4) Do you use disclosing gent to motivate the patient?

Among 50 dentists, $62 \%$ use disclosing agent to motivate the patient and $38 \%$ doesn't use disclosing agent.( Table A)

5) How much tooth paste do you recommend?

Among 50 dentists, about $28 \%$ recommend the use tooth paste of half length of brush, $12 \%$ full length of brush, $58 \%$ size of the pea, $2 \%$ size of grain of rice.

6) What do you recommend commonly for cleaning teeth?

Among 50 dentists, $46 \%$ recommend the use of brush and paste for cleaning the teeth, $24 \%$ recommend brush and paste and dental floss and $30 \%$ brush and paste, dental floss and mouth wash (fig 2)

7) Do you recommend the use of inter dental brushes during orthodontic treatment?

Among 50 dentists, about $90 \%$ recommend the use of inter dental brushes during orthodontic treatment.

$10 \%$ do not recommend the use of interdental brushes during orthodontic treatment (table A)

8) When do you recommend your patients to replace their tooth brush?

Among 50 dentists about $38 \%$ recommend their patients to replace their tooth brush once in 6 months, $46 \%$ when bristles begin to fray, $12 \%$ when the colour begin to change and $2 \%$ once in a year, $2 \%$ have no idea. (fig 3)

9) Which technique causes cervical abrasion and gingival recession?

Among 50 dentists about $8 \%$ answered charters technique, 16\% modified stillman, $64 \%$ horizontal scrub and 12 $\%$ bass technique causes cervical abrasion and gingival recession .(fig 4)

10) Which tooth brushing techniques you recommend in gingival recession and root exposure? Among 50 dentist about $18 \%$ answered bass, $44 \%$ modified stillman, $12 \%$ modified bass, $26 \%$ fones technique in gingival recession and root exposure. (fig 5)

11) What tooth brushing technique is recommended in cleaning the areas of healing wounds after periodontal surgery?

Among 50 dentists about $20 \%$ answered bass, $36 \%$ modified stillman, $42 \%$ charters and $2 \%$ horizontal scrub in cleaning the areas of healing wounds after periodontal surgery.( fig 6)

12) In type 3 embrasure, the interdental aid selected is

Among 50 dentists, $40 \%$ answered that interdental aid selected in type 3 embrasure is dental floss, $50 \%$ unitufted brushes and $10 \%$ miniature interdental aids.

\section{3) Chlorhexidine is a}

Among 50 dentists $60 \%$ answered that chlorhexidine is a Bisquanides, $12 \%$ metallic salts , $18 \%$ quaternary compounds, $10 \%$ phenolic compounds.

14) The desensitizing agent used in tooth paste is

Among 50 dentists . About $66 \%$ answered that strontium salt is the desensitizing agent used in tooth paste, $8 \%$ carboxy methyl cellulose, $16 \%$ benzoic acid, $10 \%$ calcium carbonate.

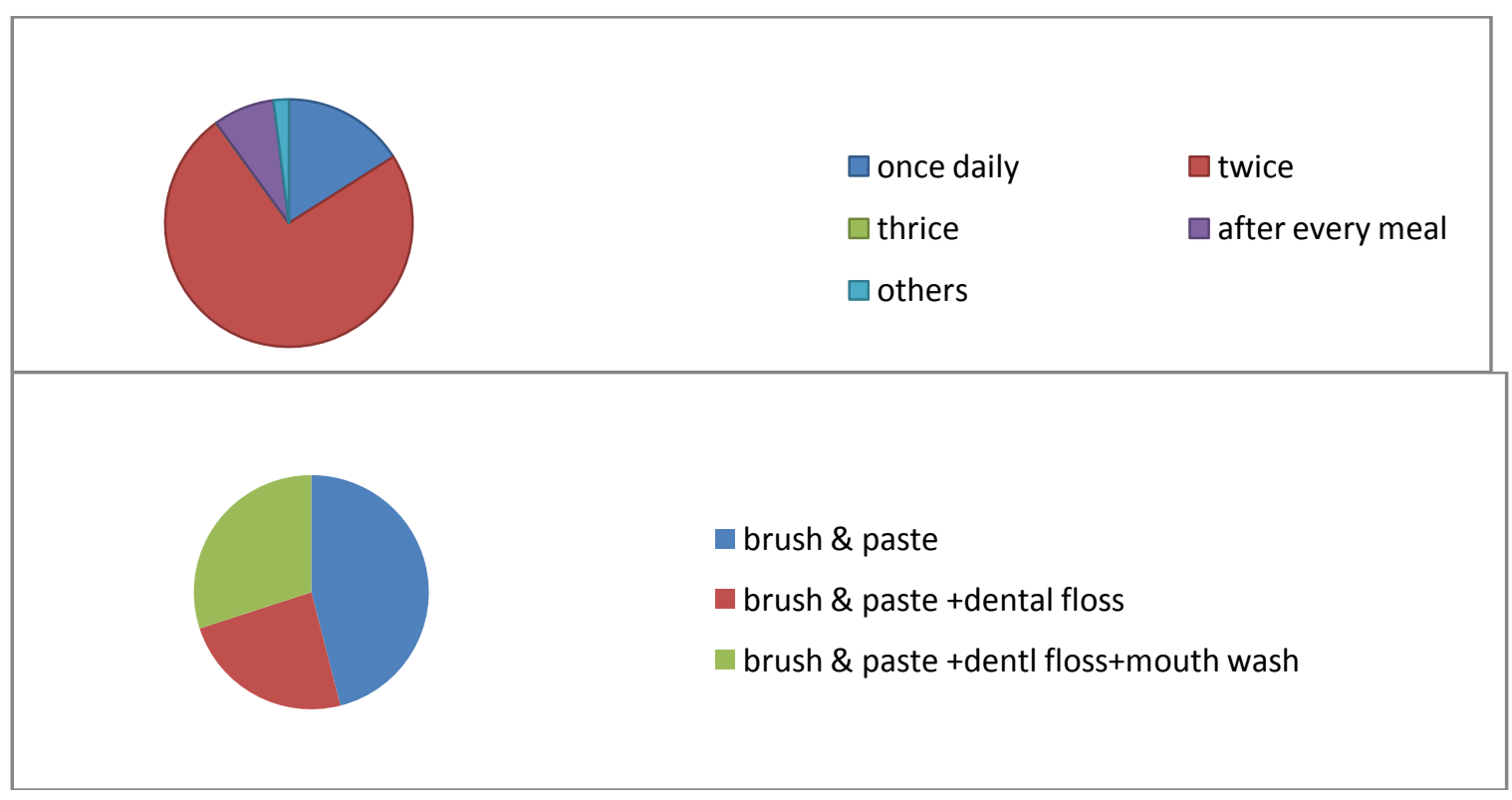




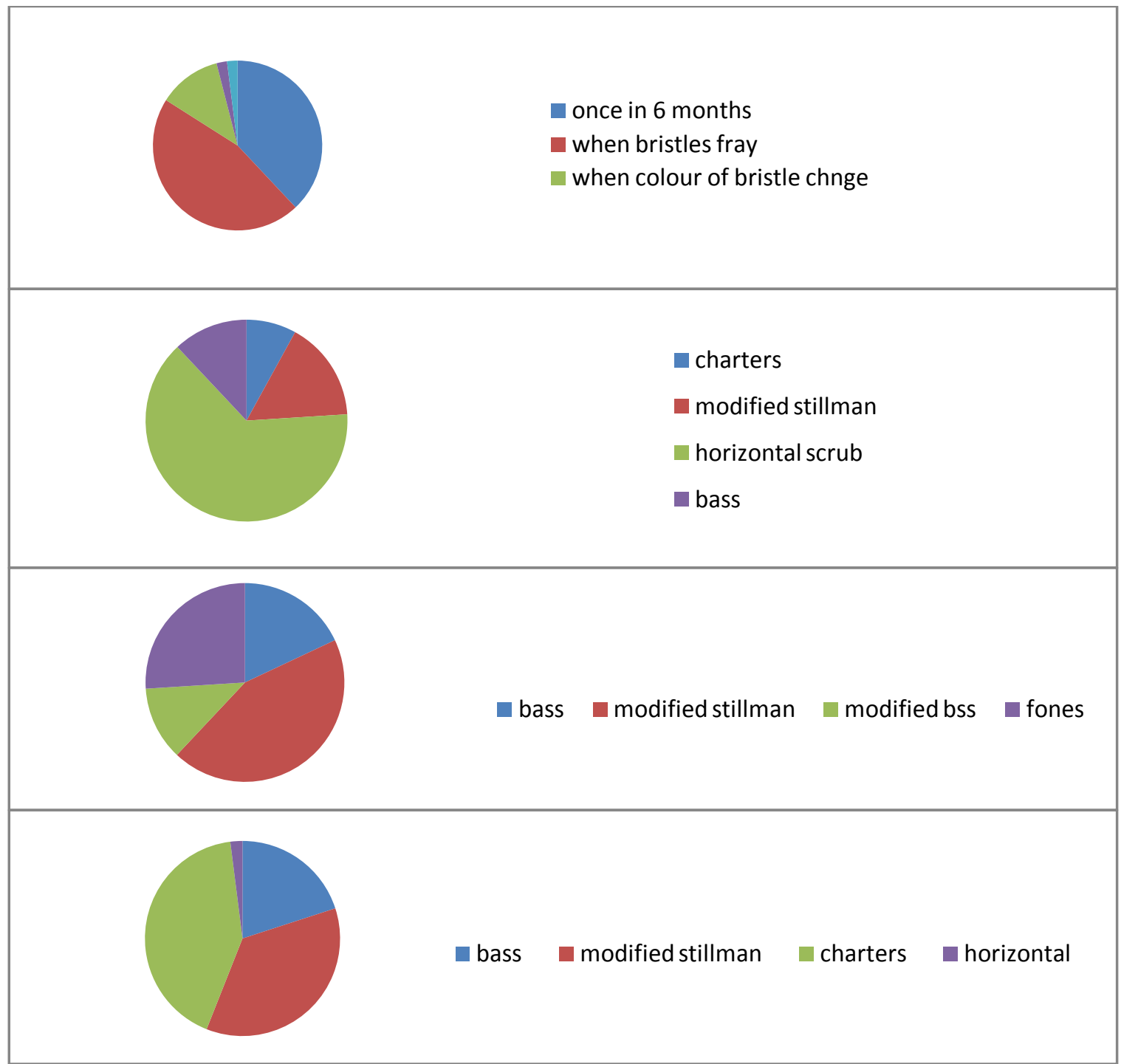

RESULTS: fig 1, fig 2, fig 3, fig 4, fig 5, fig 6

TABLE: A

\begin{tabular}{|c|l|c|c|}
\hline s.no & questions & responses \\
\hline \multirow{2}{*}{$\begin{array}{l}\text { Do you demonstrate your patients to use } \\
\text { dental floss? }\end{array}$} & yes & no \\
\cline { 3 - 4 } & $\begin{array}{l}\text { Do you use disclosing gent to motivate } \\
\text { the patient? }\end{array}$ & $62 \%$ & $18 \%$ \\
\hline 3. & $\begin{array}{l}\text { Do you recommend the use of inter } \\
\text { dental brushes during orthodontic } \\
\text { treatment? }\end{array}$ & $90 \%$ & $38 \%$ \\
\hline
\end{tabular}

\section{Discussion:}

The present study has investigated the knowledge of oral hygiene aids among dentists. Professional plaque removal and regular follow- up combined with patient oral hygiene instructions can minimize the level of gingival inflammation and swelling ${ }^{9}$

In our study about $16 \%$ recommends the cleaning of teeth once daily, $74 \%$ advise to brush twice, $8 \%$ recommend cleaning after every meals, $2 \%$ prefer others. $26 \%$ recommends brushing in the morning, $4 \%$ in the evening and $70 \%$ in the morning and night. Kressin et al (Kressin, et al. 2003) ${ }^{3}$ evaluated the effect of oral hygiene practices on tooth retention in a longitudinal study with a 26- year follow up. They observed that consistent brushing (more than once a day ) resulted in $49 \%$ reduction of risk of tooth loss compared with participants without consistent hygiene habits. A recommendation to brush twice daily should be considered (Echeverria et al 1987) ${ }^{4}$ 
About $82 \%$ demonstrate their patients to use dental floss, $18 \%$ does not demonstrate the use of dental floss. Several studies have shown the usefulness of regular dental flossing for removal of inter dental plaque and calculus ( Bauroth et al, 2003; Bellamy et al,2004) 5,6

$62 \%$ use disclosing agent to motivate the patient and $38 \%$ doesn't use disclosing agent. About $28 \%$ recommend the use tooth paste of half length of brush, $12 \%$ full length of brush, $58 \%$ size of the pea, $2 \%$ size of grain of rice. $46 \%$ recommend the use of brush and paste for cleaning the teeth, $24 \%$ recommend brush and paste and dental floss and $30 \%$ brush and paste, dental floss and mouth wash. Both American dental association and British dental association recommend the use of flossing in addition to brushing .

About $90 \%$ recommend the use of inter dental brushes during orthodontic treatment. $10 \%$ do not recommend the use of interdental brushes during orthodontic treatment. About $38 \%$ recommend their patients to replace their tooth brush once in 6 months, $46 \%$ when bristles begin to fray, $12 \%$ when the colour begin to change and $2 \%$ once in a year, $2 \%$ have no idea.

About $8 \%$ answered charters technique, $16 \%$ modified stillman, $64 \%$ horizontal scrub and $12 \%$ bass technique causes cervical abrasion and gingival recession. About $18 \%$ answered bass, $44 \%$ modified stillman, $12 \%$ modified bass, $26 \%$ Fones technique in gingival recession and root exposure.

About $20 \%$ answered bass, $36 \%$ modified stillman, $42 \%$ charters and $2 \%$ horizontal scrub in cleaning the areas of healing wounds after periodontal surgery. $40 \%$ answered that interdental aid selected in type 3 embrasure is dental floss, $50 \%$ Unitufted brushes and $10 \%$ miniature interdental aids.

$60 \%$ answered that chlorhexidine is a Bisquanides, $12 \%$ metallic salts, $18 \%$ quaternary compounds, $10 \%$ phenolic compounds. When physical plaque removal is not effective enough, adjuvant chemotherapeutic agent may play a role in controlling plaque levels. A chlorhexidine containing mouthwash is usually prescribed for a fixed period of time.

About $66 \%$ answered that strontium salt is the desensitizing agent used in tooth paste, $8 \%$ carboxy methyl cellulose, $16 \%$ benzoic acid, $10 \%$ calcium carbonate.

\section{Conclusion:}

The present Study showed the awareness and knowledge of oral hygiene aids among dental professionals. It should be noted that each patient through assessment, discussion and consent, will require oral hygiene instructions and oral hygiene aids to be tailored to their own needs. Proper oral hygiene with proper usage of oral hygiene aids will help to reduce the dental diseases.

\section{References}

[1]. Offenbacher,s Periodontal disease: pathogenesis, Ann Periodontal $1: 821-878 .,(1996)$

[2]. Schüz, B., Sniehotta, F. F., Wiedemann, A., \& Seem ann, R. (2006). Adherence to a daily flossing regimen in university students: effects of planning when, where, how and what to do in the face of barriers. Journal of Clinical Periodontology, 33(9), 612-619.

[3]. Kressin, et al. 2003, J dent research 82(3) 223-227, 2003

[4]. Echeverria, J.J., Manau, C. \& Tejerina, J.M., (1987) [fundamentals of periodontal treatment]. Arch Odonto Estomatol 3: 359-364.

[5]. Bauroth, K., Charles, C. H., Mankodi, S. M., Simmons, K., Zhao, Q. \& Kumar, L. D. (2003). The efficacy of an essential oil antiseptic mouthrinse vs. dental floss in controlling interproxi-mal gingivitis: a comparative study. Journal of the American Dental Association, 134, 359-365.

[6]. Bellamy, P., Barlow, A., Puri, G., Wright, K. I., Mussett, A. \& Zhou, X. (2004). A new in vivo interdental sampling method comparing a daily flossing regime versus a manual brush control. Journal of Clinical Dentistry, 15, 59-65

[7]. $\quad$ Lightner, L.M.,Leary,J.T.,Drake,R.B.,Chrump,P.P.\&Allen, M.F.,(1971) preventive periodontic treatment procedure:results over 46 months.J periotontal 42:555-561.

[8]. lang ,N.P.,Cumming,B.R.\&LOE,H.,(1973)Toothbrushing frequency as it to relates to plaque development and gingival health.J Periodontal 44: 396-405

[9]. Tseveenjav, B., Vehkalahti, M. \&Murutomaa, H.,(2002) Preventive practice of mongolian dental students . Eur J Dent Educ 6:7478.

[10]. Tseveenjav, B., Vehkalahti, M.\& Murtomaa, H., (2004) Oral health and its determinants among Mongolian dentists. Acta Odontol Scand 62:1-6.steenbergen, T.J., Van der veld

[11]. Christou, V., Timmerman, M.F., Van der Velden, U. \& Van der Weijden, F.A.,(1998) Comparison of different approaches of interdental oral hygiene: Interdental cleaning methods: A randomized controlled trial. J Periodontal 77: 1421-1429. 